\title{
Variations in Rectal Volumes and Dosimetry Values Including NTCP due to Interfractional Variability When Administering 2D-Based IG-IMRT for Prostate Cancer
}

\author{
Takashi Hanada, ${ }^{1,2}$ Yutaka Shiraishi, ${ }^{1}$ Toshio Ohashi, ${ }^{1}$ Junichi Fukada, ${ }^{1}$ \\ Tomoki Tanaka, ${ }^{1}$ Atsunori Yorozu, ${ }^{2}$ and Naoyuki Shigematsu ${ }^{1}$ \\ ${ }^{1}$ Department of Radiology, School of Medicine, Keio University, Shinanomachi 35, Shinjuku-ku, Tokyo 160-8582, Japan \\ ${ }^{2}$ Department of Radiology, Tokyo Medical Center, National Hospital Organization, Higashigaoka 2-5-1, Meguro-ku, \\ Tokyo 152-8902, Japan \\ Correspondence should be addressed to Takashi Hanada; thanada@rad.med.keio.ac.jp
}

Received 11 January 2014; Revised 2 July 2014; Accepted 2 July 2014; Published 15 July 2014

Academic Editor: Carlos A. Perez

Copyright (c) 2014 Takashi Hanada et al. This is an open access article distributed under the Creative Commons Attribution License, which permits unrestricted use, distribution, and reproduction in any medium, provided the original work is properly cited.

\begin{abstract}
We estimated variations in rectal volumes and dosimetry values including NTCP with interfractional motion during prostate IGIMRT. Rectal volumes, DVH parameters, and NTCPs of 20 patients were analyzed. For this patient population, the median (range) volume on the initial plan for the rectum was $45.6 \mathrm{cc}$ (31.3-82.0), showing on-treatment spread around the initial prediction based on the initial plan. DVH parameters of on-treatment CBCT analyses showed systematic regularity shift from the prediction based on the initial plan. Using the Lyman-Kutcher-Burman model, NTCPs of predicted late rectal bleeding toxicity of rectal grade $\geq$ 2 (RTOG) and the QUANTEC update rectal toxicity for the prediction based on the initial plan were $0.09 \%(0.02-0.24)$ and $0.02 \%(0.00-0.07)$, respectively, with NTCPs from on-treatment CBCT analyses being 0.35\% (0.01-6.16) and 0.12\% (0.00-4.11), respectively. Using the relative seriality model, for grade $\geq 2$ bleeding rectal toxicity, NTCP of the prediction based on the initial plan was $0.64 \%(0.15-1.22)$ versus $1.48 \%(0.18-7.66)$ for on-treatment CBCT analysis. Interfraction variations in rectal volumes occur in all patients due to physiological changes. Thus, rectal assessment during 2D-based IG-IMRT using NTCP models has the potential to provide useful and practical dosimetric verification.
\end{abstract}

\section{Introduction}

Intensity modulated radiotherapy (IMRT) allows successful delivery of escalated dose radiation to the tumor target with high precision, while simultaneously sparing sensitive adjacent tissues. This irradiation technique is adequate for treatment of prostate cancer, as increasing the dose to the prostate achieves better local control, but there is a risk of increased late toxicity [1]. The success of IMRT is related to the ability to accurately target the prostate during treatment. Several factors influence this ability, such as the accuracy of organ delineation, adequate patient immobilization, and intrafraction and interfraction organ motions. Because the treatment plan is normally based on treatment planning computed tomography (CT) images, these errors could result in the dose distribution delivered being different from that planned [2]. This may result in insufficient dose coverage to the prostate tumor volume, that is, less than that prescribed, and thereby decrease the tumor control rate. On the other hand, if the organ at risk is not at the same position as in the planning CT image, there is a potential of delivering an excessive dose possibly resulting in more serious complications.

Delivered doses can only be fully assessed using CT scans acquired at the time of treatment. Accurate interfractional patient repositioning before prostate radiotherapy has become possible in this era of image-guided radiotherapy with several in-room systems [6-9]. The KV/MV cone beam $\mathrm{CT}$ (CBCT) technique is one of these methods, allowing ontreatment acquisition of 3D images with excellent bony and reasonable soft-tissue definition, at acceptably low radiation doses [8-10]. Information can therefore be acquired for treatment set-up verification, including organ positioning, 
such that the volume treated and the daily delivered dose may be accurately calculated [11, 12].

Late rectal bleeding is one of the most important late toxicities of radiotherapy for prostate cancer as it can have a major impact on the patient's quality of life. Thus, separate doses should be delivered to the rectum during the treatment period. Changes to rectal dimensions due to rectal motions may have a substantial impact on excessive normal tissue toxicity in the rectum. Hellebust et al. reported variations in rectal doses given to patients receiving fractionated high dose rate gynecological brachytherapy using CT-based 3D treatment planning and dose volume histograms (DVH) [13]. For linear accelerator treatments, Sripadam et al. acquired CBCT scans for 15 patients with bony anatomy based setups and noted differences in rectal doses from the treatment plan, with rectal volumes being both smaller and larger than calculated averages [11]. However, it is difficult to devise an index, represented by doses or DVH parameters, allowing intuitive prediction of complications.

The possibility of combining this information in one parameter reliably expressing the probability of late rectal bleeding, as with the normal tissue complication probability (NTCP), is very attractive [14]. In this study, variations in rectal volumes and dosimetry values including NTCP of interfractional motion during prostate image-guided IMRT (IGIMRT) using CBCT were estimated to assess their usefulness for evaluating late complications. On-treatment verification CBCT images were used to study the effects of rectal motion during 2D-based IG-IMRT prostate radiotherapy.

\section{Materials and Methods}

2.1. Patient Data. Twenty patients with clinically localized stage T1c-T3b prostate carcinoma were recruited, without preselection, between January 2010 and March 2014. The median patient age was 72 years, and the median prostate specific antigen level and Gleason score were $8.5 \mathrm{ng} \cdot \mathrm{mL}^{-1}$ and 7.5, respectively. All patients completed their planned treatment course. There were no preparations for these patients after those of the bladder and bowel protocol, aimed at an empty rectum and full bladder, either before the initial planning scan or at any time during the treatment. For treatment planning, patients were scanned on a large bore fan-beam CT scanner with a $2.0 \mathrm{~mm}$ slice thickness in the supine position.

2.2. On-Treatment CBCT Acquisition. At each treatment session, the patient was placed in the appropriate position using simulation markers and alignment lasers, and orthogonal $\mathrm{kV}$ images were then acquired. These images were used online to match the pelvic bone positions to the initial plan reference images and the patient was moved to the aligned position. No tolerance for shifting was applied, such that all daily measured set-up errors were corrected with online bony 2D matching. Patients remained supine on a flat carbon fiber couch top with vacuum cushions. CBCTs were scheduled after the initial patient set-up to confirm the interfractional motions of the prostate. Acquired images were subsequently analyzed on a weekly basis during the treatment course (8 CBCTs/patient).
2.3. Dose Calculation and Assessment. The rectal volume was contoured from anal verge to the rectosigmoid junction. The planning treatment volume (PTV) was determined using the $\mathrm{XiO}$ planning system (Elekta, Stockholm, Sweden) for the scan from the initial planning $\mathrm{CT}$ and each on-treatment $\mathrm{CBCT}$. All scans used a margin of $6 \mathrm{~mm}$ posterior and $0.7-$ $1 \mathrm{~cm}$ in other directions around the clinical target volume to determine the PTV. The PTV was defined as the prostate alone to which a dose of $74 \mathrm{~Gy}$ in 37 fractions had been prescribed for 3 patients and the prostate including seminal vesicles to which a dose of $78 \mathrm{~Gy}$ in 39 fractions had been prescribed for 10 patients and $80 \mathrm{~Gy}$ in 40 fractions for 7 patients. Energy equivalent to an X-ray dose of $10 \mathrm{MV}$ was used for treatment, normalized at the PTV for 95\% isodose coverage. This initial plan was then applied to all CBCTs for each patient to calculate the daily dose delivered. Doses calculated using Hounsfield units (HU) from CBCTs have errors due to inaccuracies inherent to the $\mathrm{HU}$ values. Since CBCT images suffer from an increased contribution of scatter, resulting in reconstruction errors in these images, thereby leading to dose errors, comparisons were made by not adopting heterogeneity corrections of dose calculations for either the initial planning or the CBCT images, allowing assessment of the actual effects of rectal motions.

For each patient, the on-treatment DVHs for the rectum were exported and compared with the initial plan DVH. Rectal volume and DVH parameters, that is, $D_{\mathrm{MAX}}, D_{2 \%}$, $D_{\text {MEAN }}, D_{2 c c}$, and $D_{0.1 c c}$, were calculated and analyzed. Here, $D_{\mathrm{MAX}}$ and $D_{\mathrm{MEAN}}$ are the maximum and mean dose deposited in rectum, and $D_{X}$ is the minimal dose deposited in $X \%$ or $X$ cc of the rectal volume.

NTCPs were analyzed using the Lyman-Kutcher-Burman (LKB) model and the relative seriality (RS) model. To account for variations in dose per fraction in different subvolumes of the rectum with changes in fractionation schedules, the total physical dose corresponding to each DVH bin, $D_{i}$, was converted into an isoeffective dose in $2 \mathrm{~Gy}$ fractions using the following equation [15]:

$$
\mathrm{LQED}_{i}=D_{i} \cdot \frac{1+\left(\left(D_{i} / N\right) /(\alpha / \beta)\right)}{1+(2 /(\alpha / \beta))},
$$

where $N$ is the number of fractions. According to the literature, radiation responses are generally determined for $2 \mathrm{~Gy}$ fractions, making it good practice to utilize LQED2 rather than the physical dose in calculations of NTCP [15]. The LKB model is defined as follows [15]:

$$
\mathrm{NTCP}_{\mathrm{LKB}}=\frac{1}{\sqrt{2 \cdot \pi}} \int_{-\infty}^{t} e^{-t^{\prime 2} / 2} \cdot d t^{\prime},
$$

where

$$
\begin{gathered}
t=\frac{D_{\mathrm{eff}}-T D_{50}}{m \cdot T D_{50}}, \\
D_{\mathrm{eff}}=\left(\sum_{i} v_{i} \cdot \operatorname{LQED} 2_{i}{ }^{1 / n}\right)^{n} .
\end{gathered}
$$

$D_{\text {eff }}$ is the dose that, if given uniformly to the entire volume, will lead to the same NTCP as the actual nonuniform dose 
TABLE 1: The default parameter values of the LKB and RS models employed in this study for the rectum.

\begin{tabular}{lccc}
\hline Parameters & & LKB [3,4] & RS [5] \\
\hline$\alpha / \beta$ ratio & - & 3 Gy & 0.49 \\
$s$ & 0.14 & - & -13 \\
$m$ & 0.08 & 0.09 & - \\
$n$ & 78 Gy for rectal grade $\geq 2$ (RTOG) & 76.9 Gy for combined grade $\geq 2$ rectal & 83.1 Gy for rectal grade $\geq 2$ bleeding \\
$D_{50}$ & & toxicity (QUANTEC update) & \\
\hline
\end{tabular}

distribution, $\mathrm{TD}_{50}$ is the uniform dose given to the entire organ that results in a $50 \%$ complication risk, $m$ is a measure of the slope of the sigmoid curve, $n$ is the volume effect parameter, and $v_{i}$ is the fractional organ volume receiving a dose $D_{i}$. The RS model is based on Poisson statistics and accounts for the architecture of the organ through the $s$ parameter. The RS model is derived from the ratio of serial subunits to all subunits in the organ. For a heterogeneous dose distribution the complication probability is given by [15]

$$
\mathrm{NTCP}_{\mathrm{RS}}=\left\{1-\prod_{i}\left[1-P\left(\mathrm{LQED}_{i}\right)^{s}\right]^{v_{i}}\right\}^{1 / s},
$$

where $P\left(\mathrm{LQED} 2_{i}\right)$ is the complication rate derived from the Poisson model. In-house software was used to estimate rectal NTCP using the LKB model and the RS model, and the model parameters used for the calculations for each rectum are summarized in Table 1 [3-5].

\section{Results}

3.1. Rectum Volumes. A list of volumetric values of the rectum for prostate radiation treatment with IG-IMRT by online bony 2D matching techniques is presented in Table 2 . These values from the initial planning CT and analyses from on-treatment CBCTs are compared. Across the patient population, the median (range) volume on the planning CT for the rectum was $45.6 \mathrm{cc}(31.3-82.0)$. Rectal volumes show the spread of on-treatment plan values around the prediction based on the initial plan. In several patients, the on-treatment rectal volume is more than twice that of the initial plan.

3.2. DVH Parameters. Differences in DVH parameters, that is, $D_{\text {MAX }}, D_{2 \%}, D_{\text {MEAN }}, D_{2 c c}$, and $D_{0.1 c c}$, are summarized in Table 3. In this study, for comparison of two values, results are given in terms of relative differences. The relative differences in $D_{X}\left(\Delta D_{X}\right)$ between the prediction based on the initial plan $\left(D_{X, \text { ip }}\right)$ and on-treatment CBCT analyses $\left(D_{X, \mathrm{CBCT}}\right)$ are given as follows:

$$
\Delta D_{X}(\mathrm{~Gy})=D_{X, \mathrm{CBCT}}-D_{X, \mathrm{ip}} .
$$

Differences in DVH parameters show systematic regularity shift from the initial plan, since 17 patients had median $D_{X}$ based on the on-treatment CBCT analyses higher than the prediction based on the initial plan, while 2 patients had lower $D_{X}$. One patient (patient 13 ) had median $D_{\text {MAX }}$ and $D_{\text {MEAN }}$
TABLE 2: Rectal interfraction volume changes.

\begin{tabular}{lcc}
\hline Patient number & \multicolumn{2}{c}{ Volume (cc) } \\
& Initial plan & $\begin{array}{c}\text { CBCTs } \\
\text { Median (range) }\end{array}$ \\
\hline 1 & 41.8 & $47.5(35.0-82.7)$ \\
2 & 82.0 & $74.4(60.1-99.9)$ \\
3 & 43.1 & $53.7(38.9-77.0)$ \\
4 & 38.7 & $40.8(36.3-89.2)$ \\
5 & 31.3 & $32.3(25.8-36.9)$ \\
6 & 43.2 & $42.2(39.0-108.3)$ \\
7 & 39.8 & $36.9(30.8-89.7)$ \\
8 & 54.3 & $52.6(45.5-82.2)$ \\
9 & 65.8 & $56.0(51.9-83.4)$ \\
10 & 50.6 & $42.5(35.6-77.7)$ \\
11 & 69.3 & $62.9(48.9-96.3)$ \\
12 & 62.3 & $53.7(43.6-83.8)$ \\
13 & 50.4 & $48.4(43.8-70.3)$ \\
14 & 47.3 & $39.6(32.4-41.7)$ \\
15 & 33.1 & $34.7(29.1-46.3)$ \\
16 & 45.7 & $32.5(30.7-55.8)$ \\
17 & 42.4 & $44.7(40.0-46.6)$ \\
18 & 45.6 & $40.1(37.3-48.8)$ \\
19 & 51.2 & $49.9(40.2-92.9)$ \\
20 & 36.8 & $44.7(38.1-60.5)$ \\
\hline & &
\end{tabular}

from on-treatment $\mathrm{CBCT}$ analyses higher than the prediction based on the initial plan, while other $D_{X}$, that is, $D_{2 \%}, D_{2 c c}$, and $D_{0.1 \mathrm{cc}}$, were lower. To illustrate individual patient data, Figure 1 shows the spread of $D_{2 \%}$ for on-treatment values around the prediction based on the initial plan, as an example. None of the patients show any recognizable variation pattern.

3.3. Rectal NTCP. Table 4 shows NTCP of late rectal bleeding as predicted from the initial plan and on-treatment CBCT analyses in 2D-based IG-IMRT. Calculations, made with the $\mathrm{LKB}$ model and the RS model, indicate the risks of rectal complications based on the on-treatment CBCT analyses to be higher than those predicted based on the initial plan. Figures 2 to 4 show the details of NTCP from on-treatment CBCT analyses, spread around the prediction based on the initial plan, in individual patients. None of the patients showed any recognizable pattern in variation or in $D_{2 \%}$, with some having a broad range of values, while in others the spread of NTCPs was within a narrow range. 
TABLE 3: Summary of differences in DVH parameters presented as relative differences. These values are relative differences represented as the median (range) defined in (5) (see text).

\begin{tabular}{|c|c|c|c|c|c|}
\hline Patient number & $D_{\mathrm{MAX}}(\mathrm{Gy})$ & $D_{2 \%}(\mathrm{~Gy})$ & $D_{\text {MEAN }}(\mathrm{Gy})$ & $D_{2 c c}(\mathrm{~Gy})$ & $D_{0.1 c c}(\mathrm{~Gy})$ \\
\hline 1 & $\begin{array}{c}3.2 \\
(-0.4-3.5)\end{array}$ & $\begin{array}{c}2.6 \\
(-1.7-4.3)\end{array}$ & $\begin{array}{c}2.6 \\
(0.6-12.5)\end{array}$ & $\begin{array}{c}3.9 \\
(-1.9-6.5)\end{array}$ & $\begin{array}{c}2.1 \\
(-0.7-3.8)\end{array}$ \\
\hline 2 & $\begin{array}{c}2.9 \\
(1.7-3.4)\end{array}$ & $\begin{array}{c}4.2 \\
(2.1-6.3)\end{array}$ & $\begin{array}{c}2.2 \\
(-0.6-6.1)\end{array}$ & $\begin{array}{c}3.4 \\
(1.2-6.9)\end{array}$ & $\begin{array}{c}2.8 \\
(1.3-3.9)\end{array}$ \\
\hline 3 & $\begin{array}{c}3.0 \\
(2.5-3.4)\end{array}$ & $\begin{array}{c}5.9 \\
(3.1-6.4)\end{array}$ & $\begin{array}{c}7.5 \\
(2.0-11.8)\end{array}$ & $\begin{array}{c}8.0 \\
(3.7-8.9)\end{array}$ & $\begin{array}{c}4.4 \\
(2.9-4.7)\end{array}$ \\
\hline 4 & $\begin{array}{c}-3.0 \\
(-12.5-2.4)\end{array}$ & $\begin{array}{c}-4.6 \\
(-15.8-4.3)\end{array}$ & $\begin{array}{c}-2.0 \\
(-6.0-5.0)\end{array}$ & $\begin{array}{c}-5.0 \\
(-16.9-7.0)\end{array}$ & $\begin{array}{c}-3.3 \\
(-14.2-3.5)\end{array}$ \\
\hline 5 & $\begin{array}{c}1.8 \\
(-2.2-2.7)\end{array}$ & $\begin{array}{c}3.7 \\
(-2.6-6.3)\end{array}$ & $\begin{array}{c}3.6 \\
(-1.2-5.9)\end{array}$ & $\begin{array}{c}6.6 \\
(-3.5-10.8)\end{array}$ & $\begin{array}{c}2.8 \\
(-2.3-4.3)\end{array}$ \\
\hline 6 & $\begin{array}{c}2.4 \\
(1.5-2.6)\end{array}$ & $\begin{array}{c}4.4 \\
(0.2-4.9)\end{array}$ & $\begin{array}{c}6.1 \\
(-0.3-9.9)\end{array}$ & $\begin{array}{c}6.0 \\
(1.6-7.9)\end{array}$ & $\begin{array}{c}3.3 \\
(1.6-3.7)\end{array}$ \\
\hline 7 & $\begin{array}{c}2.5 \\
(-10.5-4.1)\end{array}$ & $\begin{array}{c}2.8 \\
(-14.8-6.1)\end{array}$ & $\begin{array}{c}1.1 \\
(-7.9-13.0)\end{array}$ & $\begin{array}{c}2.2 \\
(-18.4-8.3)\end{array}$ & $\begin{array}{c}2.8 \\
(-11.7-5.0)\end{array}$ \\
\hline 8 & $\begin{array}{c}0.8 \\
(0.2-0.8)\end{array}$ & $\begin{array}{c}4.4 \\
(2.3-5.4)\end{array}$ & $\begin{array}{c}7.6 \\
(4.0-14.0)\end{array}$ & $\begin{array}{c}6.2 \\
(3.2-8.3)\end{array}$ & $\begin{array}{c}1.8 \\
(0.7-2.1)\end{array}$ \\
\hline 9 & $\begin{array}{c}1.4 \\
(0.3-3.2)\end{array}$ & $\begin{array}{c}5.6 \\
(4.4-6.7)\end{array}$ & $\begin{array}{c}5.8 \\
(3.6-12.4)\end{array}$ & $\begin{array}{c}6.6 \\
(5.0-8.2)\end{array}$ & $\begin{array}{c}2.6 \\
(1.7-3.7)\end{array}$ \\
\hline 10 & $\begin{array}{c}0.8 \\
(-1.5-2.1)\end{array}$ & $\begin{array}{c}3.5 \\
(-2.2-5.8)\end{array}$ & $\begin{array}{c}8.4 \\
(0.0-14.4)\end{array}$ & $\begin{array}{c}4.3 \\
(-2.7-8.1)\end{array}$ & $\begin{array}{c}2.0 \\
(-1.3-3.2)\end{array}$ \\
\hline 11 & $\begin{array}{c}1.6 \\
(0.7-2.9)\end{array}$ & $\begin{array}{c}2.1 \\
(0.4-8.2)\end{array}$ & $\begin{array}{c}0.0 \\
(-2.6-4.5)\end{array}$ & $\begin{array}{c}1.8 \\
(-3.3-9.6)\end{array}$ & $\begin{array}{c}1.9 \\
(0.4-3.8)\end{array}$ \\
\hline 12 & $\begin{array}{c}4.2 \\
(0.6-4.5)\end{array}$ & $\begin{array}{c}6.8 \\
(3.9-10.4)\end{array}$ & $\begin{array}{c}5.4 \\
(0.2-7.1)\end{array}$ & $\begin{array}{c}6.7 \\
(-1.5-8.4)\end{array}$ & $\begin{array}{c}4.2 \\
(-0.5-5.2)\end{array}$ \\
\hline 13 & $\begin{array}{c}0.1 \\
(-1.4-1.6)\end{array}$ & $\begin{array}{c}-0.8 \\
(-2.6-3.1)\end{array}$ & $\begin{array}{c}0.4 \\
(-5.2-1.6)\end{array}$ & $\begin{array}{c}-1.4 \\
(-3.2-4.5)\end{array}$ & $\begin{array}{c}-0.2 \\
(-1.5-2.1)\end{array}$ \\
\hline 14 & $\begin{array}{c}3.4 \\
(3.2-3.4)\end{array}$ & $\begin{array}{c}5.6 \\
(5.2-5.9)\end{array}$ & $\begin{array}{c}14.6 \\
(9.9-19.2)\end{array}$ & $\begin{array}{c}7.9 \\
(6.4-8.1)\end{array}$ & $\begin{array}{c}4.0 \\
(3.5-4.1)\end{array}$ \\
\hline 15 & $\begin{array}{c}1.4 \\
(-5.3-3.9)\end{array}$ & $\begin{array}{c}0.8 \\
(-7.7-7.1)\end{array}$ & $\begin{array}{c}0.8 \\
(-6.8-14.2)\end{array}$ & $\begin{array}{c}2.7 \\
(-10.7-11.7)\end{array}$ & $\begin{array}{c}1.2 \\
(-5.6-4.8)\end{array}$ \\
\hline 16 & $\begin{array}{c}1.3 \\
(0.1-2.0)\end{array}$ & $\begin{array}{c}2.4 \\
(0.6-5.1)\end{array}$ & $\begin{array}{c}6.3 \\
(4.2-15.0)\end{array}$ & $\begin{array}{c}2.2 \\
(-0.8-7.8)\end{array}$ & $\begin{array}{c}1.7 \\
(0.3-2.9)\end{array}$ \\
\hline 17 & $\begin{array}{c}2.3 \\
(0.7-3.0)\end{array}$ & $\begin{array}{c}4.0 \\
(0.3-4.9)\end{array}$ & $\begin{array}{c}6.9 \\
(-0.9-10.2)\end{array}$ & $\begin{array}{c}5.9 \\
(-0.8-7.1)\end{array}$ & $\begin{array}{c}2.8 \\
(0.6-3.6)\end{array}$ \\
\hline 18 & $\begin{array}{c}2.3 \\
(1.3-2.4)\end{array}$ & $\begin{array}{c}3.1 \\
(1.3-3.6)\end{array}$ & $\begin{array}{c}3.5 \\
(1.8-6.1)\end{array}$ & $\begin{array}{c}3.5 \\
(1.0-4.5)\end{array}$ & $\begin{array}{c}2.5 \\
(1.1-2.9)\end{array}$ \\
\hline 19 & $\begin{array}{c}-0.7 \\
(-4.3-2.0)\end{array}$ & $\begin{array}{c}-3.1 \\
(-8.9-4.4)\end{array}$ & $\begin{array}{c}-3.8 \\
(-6.0-0.3)\end{array}$ & $\begin{array}{c}-4.1 \\
(-13.1-6.8)\end{array}$ & $\begin{array}{c}-1.6 \\
(-5.8-3.0)\end{array}$ \\
\hline 20 & $\begin{array}{c}4.8 \\
(3.4-6.0)\end{array}$ & $\begin{array}{c}7.1 \\
(2.7-9.2)\end{array}$ & $\begin{array}{c}4.4 \\
(-4.0-6.8)\end{array}$ & $\begin{array}{c}10.9 \\
(4.4-14.5)\end{array}$ & $\begin{array}{c}5.5 \\
(2.9-6.6)\end{array}$ \\
\hline
\end{tabular}

TABLE 4: Summary of NTCPs (\%) for the rectum: predictions based on the initial plan and on-treatment CBCTs analyses.

\begin{tabular}{lcr}
\hline Late rectal bleeding & Initial plan & CBCTs \\
\hline & Rectal grade $\geq 2$ (RTOG) using LKB model & $1.12 \pm 0.76$ \\
Mean \pm SD & $0.10 \pm 0.06$ & $0.35(0.01-6.16)$ \\
Median (range) & $0.09(0.02-0.24)$ & $2.29 \pm 0.83$ \\
\hline & Rectal grade $\geq 2$ (bleeding) using RS model & $1.48(0.18-7.66)$ \\
Mean \pm SD & $0.65 \pm 0.30$ & \\
Median (range) & $0.64(0.15-1.22)$ & $0.62 \pm 0.55$ \\
Mean \pm SD & Combined grade $\geq 2$ rectal toxicity (QUANTEC update) using LKB model & $0.12(0.00-4.11)$ \\
Median (range) & $0.03 \pm 0.02$ & $0.02(0.00-0.07)$ \\
\hline
\end{tabular}




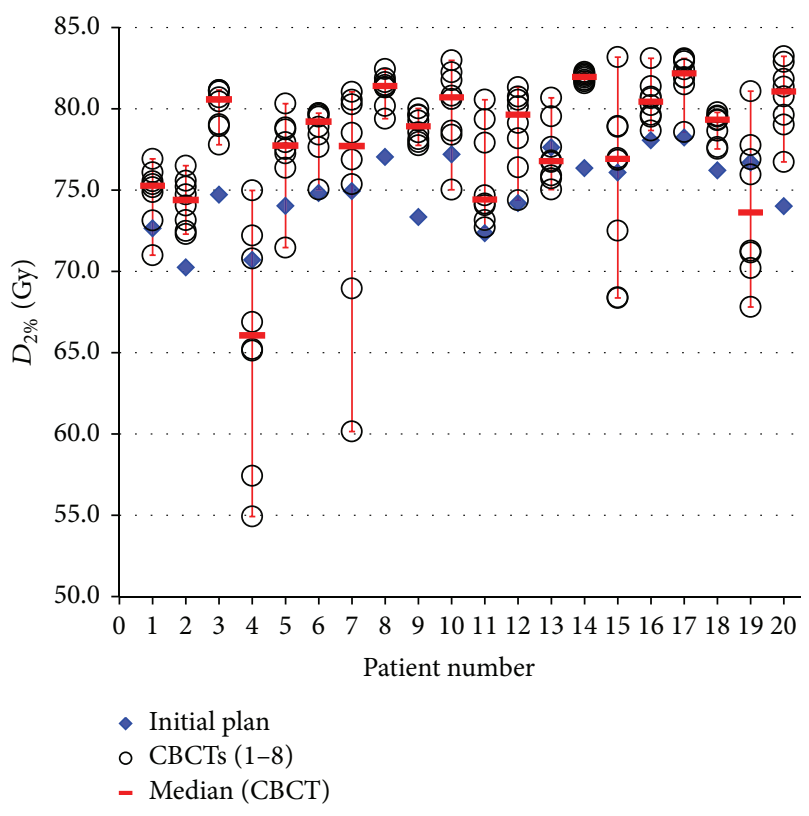

FIGURE 1: $D_{2 \%}$ for the rectum: predictions based on the initial plan and on-treatment CBCT analyses (8 CBCTs/patient).

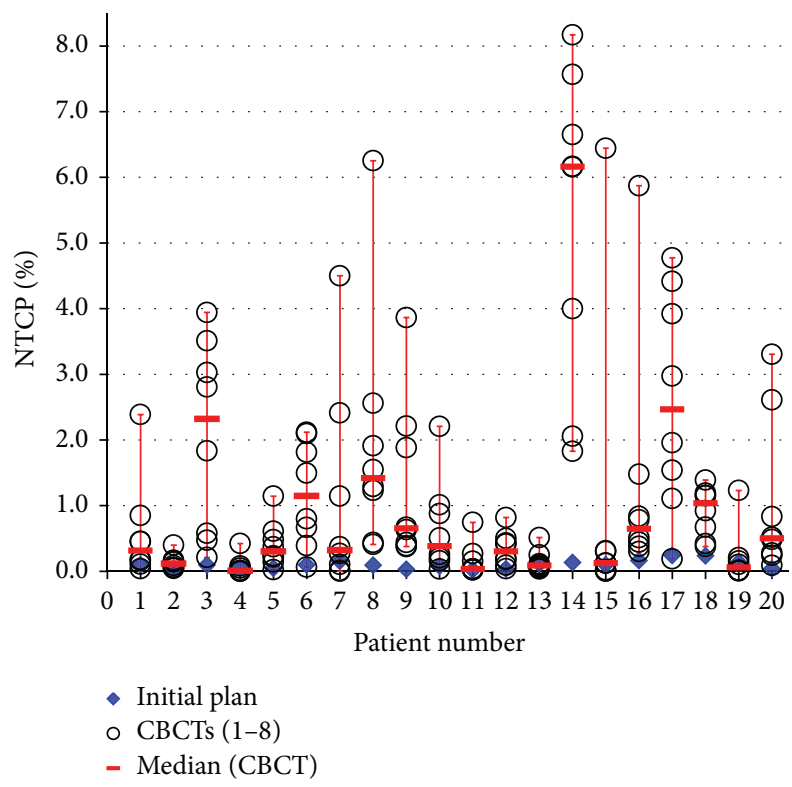

FIGURE 2: NTCPs for the rectum: predictions based on initial plan and on-treatment $\mathrm{CBCT}$ analyses (8 $\mathrm{CBCTs} /$ patient) for rectal grade $\geq 2$ (RTOG) using the LKB model.

\section{Discussion}

It is well known that, compared to $\mathrm{kV}$ CT images, CBCT images suffer from increased scatter [16]. In general, Xray scatter reduces image contrast, increases image noise, and may introduce reconstruction errors into CBCT images. In addition, the contribution of scatter is patient geometry dependent. Consequently, the CT numbers of a CBCT scan cannot simply be converted to electron density and directly used for dose recomputation, as this may lead to dose errors

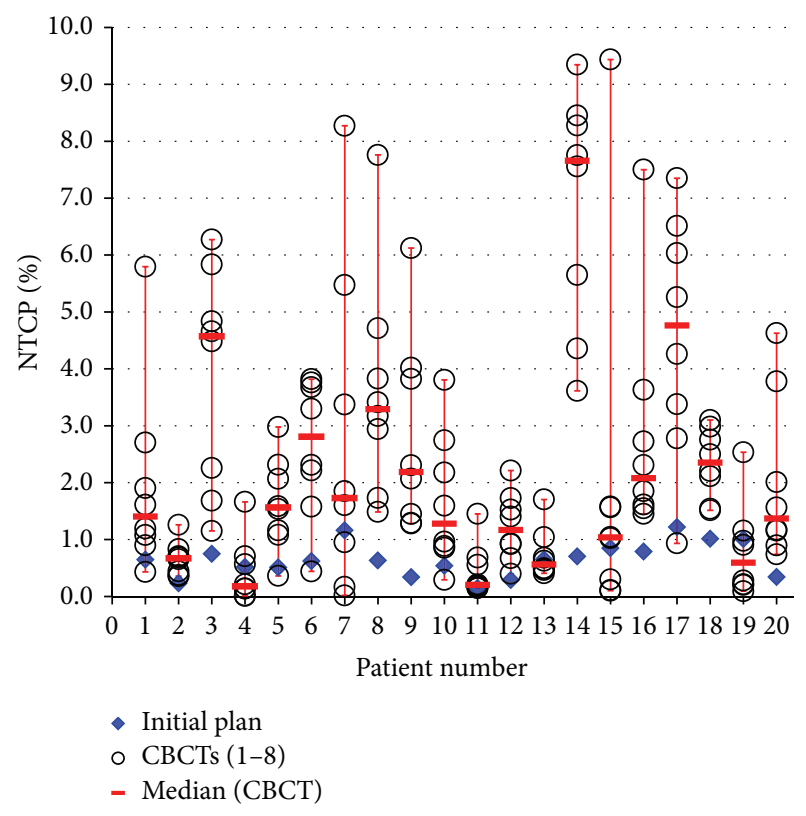

FIGURE 3: NTCPs for the rectum: predictions based on the initial plan and on-treatment CBCT analyses (8 CBCTs/patient) for rectal grade $\geq 2$ bleeding using the RS model.

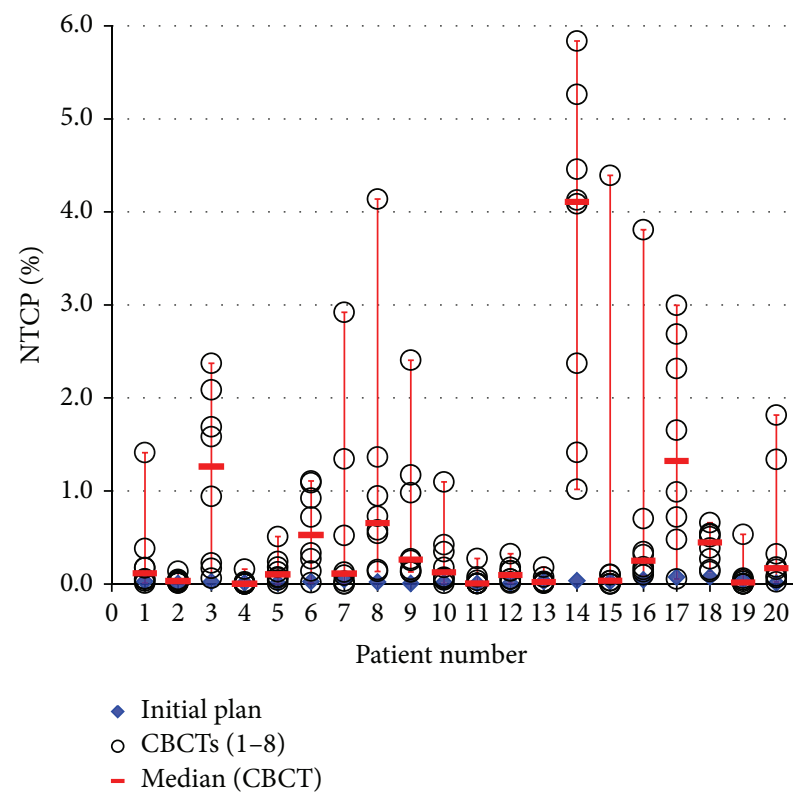

FIGURE 4: NTCPs for rectum: predictions based on the initial plan and on-treatment CBCTs analyses (8 CBCTs/patient) for combined grade $\geq 2$ rectal toxicity from the QUANTEC update using the LKB model.

$[17,18]$. Yang et al. have shown differences in the maximum critical structure dose between the results of the Monte Carlo method and the radiotherapy planning system without heterogeneity correction being negligible [19]. For these reasons, we considered using methods without heterogeneity correction for dose calculations to be appropriate for making 


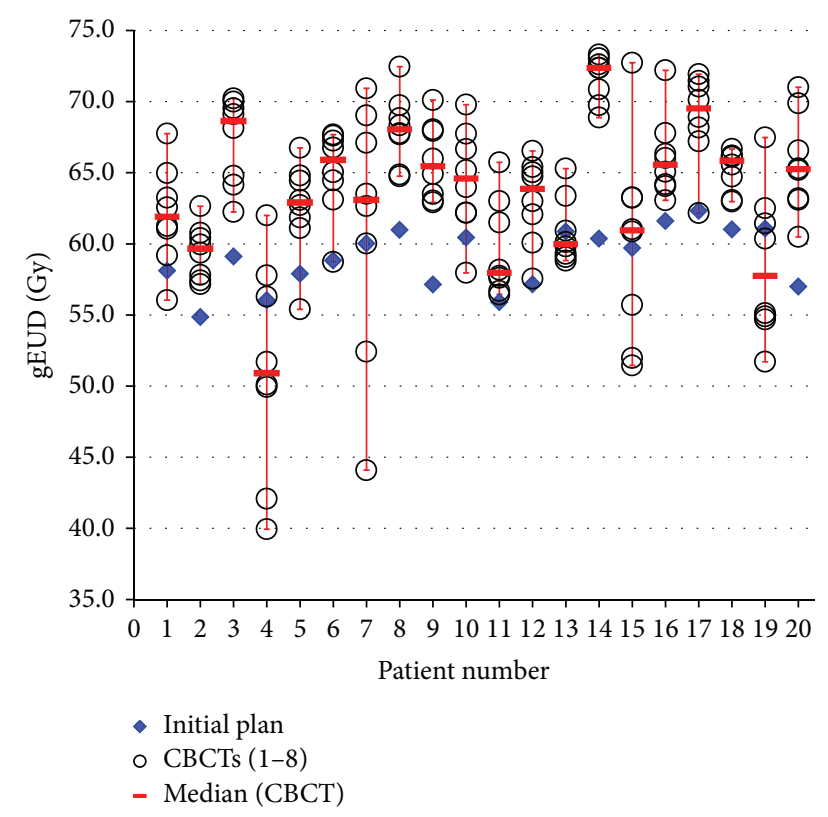

FIGURE 5: The values of gEUD for the rectum: predictions based on the initial plan and on-treatment CBCT analyses (8 CBCTs/patient).

comparisons between initial planning $\mathrm{CT}$ images and ontreatment CBCT images.

None of our patients showed consistent differences in rectal volumes (Table 2); however, the majority of patients showed systematic regularity shift in DVH parameters between the prediction based on the initial plan and ontreatment CBCT analyses (Table 3). No apparent patterns were discernible when making comparisons among patients or within individual patients, in $D_{2 \%}$ data values (Figure 1). Some patients did exhibit larger variations than others, but, overall, the variations were essentially random. In a previous study, Hatton et al. found that rectal DVH results tended to underestimate the on-treatment rectal dose, with $65 \%$ of all on-treatment plans showing higher rectal doses than predicted [12]. Their study demonstrated the generally limited effectiveness of the rectal preparation protocols used, based on CBCT scans. The study by Kupelian et al. included a rectal enema for the initial CT scan, with no specific instructions for bowel preparations prior to treatment, and found similar significant variations in rectal DVH, leading the authors to conclude that the planned dose was a poor predictor of actual doses delivered to the rectum [20]. Pawlowski et al., who proposed that variations in rectal filling are reflected in dosimetric variations, specified no rectal preparation [21]. In their study, no preparation protocol for the rectum was prescribed, resulting in randomness in the rectal filling variation, and the doses delivered to the rectum were unpredictable. Variation in rectal volumes occurs in all patients due to physiological changes. Whatever the factors underlying these rectal volume differences, the current study provides a quantitative assessment of the dosimetric impact of such variations.

Because of the daily variation in rectal filling, the actual delivered radiation doses did not match the treatment plan in the large majority of fractions, leading to the spread of NTCPs from on-treatment CBCT analyses around the prediction based on the initial plan (Figures 2, 3, and 4). In this study, 2 different NTCP models were used to assess endpoints for 3 types of late rectal bleeding. Results for the prediction of NTCP values based on the initial plan showed a tendency for underestimation of the on-treatment NTCP values, with all NTCP models, indicating that, regardless of which models or parameters are used, the same extents and tendencies are observed for all results (Table 4). Reviewing the NTCP results for individual patients, severe late rectal bleeding was rare. It should be noted that such NCTPs are assumed to represent radiation dose deliveries given to patients in a total of 37,39 , or 40 fractions, such that there is a possibility of overestimating the NTCP by on-treatment CBCT analyses. However, overestimating NTCP involving the organs at risk requires adequate attention to radiation safety, and, in addition, it is helpful to clinicians since the NTCP reliably expresses the probability of late rectal bleeding. The possibility of complications can, in fact, more easily be assessed intuitively than with physical doses or DVH parameters. Additionally, the extent of the range of $D_{2 \%}$ values for patients is not systematically shown by mapping the range of NTCPs in any currently available model. Spread of generalized equivalent uniform dose (gEUD) for on-treatment values around the prediction based on the initial plan is shown in Figure 5 for comparison, indicating similar extents and tendencies to the result of $D_{2 \%}[15,22]$. These findings confirm that it is difficult to predict late toxicity (i.e., rectal bleeding in this study) using only a physical dose index, like one of the DVH parameters and gEUD, as a predictive factor, especially for organs at risk or having parallel components. In a clinical setting and with on-treatment scans acquired during treatment, conformal plans calculated based on the on-treatment scans have the potential to provide accurate predictions of the overall physical doses delivered and to indicate NTCP. Developing the methods allowing feedback of such information to the clinician for implementation of treatment planning recalculation or for quality assurance would be useful [23]. However, the aspect of adding the process of NTCP calculations into adaptive workflows or into the evaluation phase for resimulation and replanning will need further investigation.

The 20 case studies presented herein are large enough sample to reflect the outcomes in our entire patient population. In addition, detailed examination of individual patient data allows recognition of outcomes which could easily be missed in grouped data. As our findings show, this case series was large enough to identify common issues relevant to the accuracy of the initial treatment plan dose prediction and NTCP for the rectum as compared to that actually experienced throughout the treatment course. These observations warrant further investigation.

\section{Conclusions}

Employing an online matching protocol for pelvic bone, we assessed the influences of variations in interfraction motions on rectal volumes and dosimetry values, such as 
DVH parameters and NTCP, and detected no recognizable variation patterns. This approach to assessing interfractional variability in the rectum receiving $2 \mathrm{D}$-based IG-IMRT during radiation treatment for prostate cancer using NTCP models could provide a useful and practical means of dosimetric verification and overcome limitations of the physical dose index.

\section{Conflict of Interests}

The authors declare that there is no conflict of interests regarding the publication of this paper.

\section{Acknowledgment}

The authors are grateful to Mrs. Kazuko Ogawa at Keio University School of Medicine for her support and assistance.

\section{References}

[1] M. J. Zelefsky, E. J. Levin, M. Hunt et al., "Incidence of late rectal and urinary toxicities after three-dimensional conformal radiotherapy and intensity-modulated radiotherapy for localized prostate cancer," International Journal of Radiation Oncology Biology Physics, vol. 70, no. 4, pp. 1124-1129, 2008.

[2] R. Varadhan, S. K. Hui, S. Way, and K. Nisi, "Assessing prostate, bladder and rectal doses during image guided radiation therapy-need for plan adaptation?" Journal of applied clinical medical physics, vol. 10, article 2883, no. 3, 2009.

[3] S. L. Tucker, L. Dong, W. R. Bosch et al., "Late rectal toxicity on RTOG 94-06: analysis using a mixture lyman model," International Journal of Radiation Oncology Biology Physics, vol. 78, no. 4, pp. 1253-1260, 2010.

[4] J. M. Michalski, H. Gay, A. Jackson, S. L. Tucker, and J. O. Deasy, "Radiation dose-volume effects in radiation-induced rectal injury," International Journal of Radiation Oncology Biology Physics, vol. 76, no. 3, pp. S123-S129, 2010.

[5] T. Rancati, C. Fiorino, G. Gagliardi et al., "Fitting late rectal bleeding data using different NTCP models: Results from an Italian multi-centric study (AIROPROS0101)," Radiotherapy and Oncology, vol. 73, no. 1, pp. 21-32, 2004.

[6] T. R. Willoughby, P. A. Kupelian, J. Pouliot et al., “Target localization and real-time tracking using the Calypso 4D localization system in patients with localized prostate cancer," International Journal of Radiation Oncology Biology Physics, vol. 65, no. 2, pp. 528-534, 2006.

[7] J. Boda-Heggemann, F. M. Köhler, B. Küpper et al., "Accuracy of ultrasound-based (BAT) prostate-repositioning: a threedimensional on-line fiducial-based assessment with cone-beam computed tomography," International Journal of Radiation Oncology, Biology, Physics, vol. 70, no. 4, pp. 1247-1255, 2008.

[8] C. A. McBain, A. M. Henry, J. Sykes et al., "X-ray volumetric imaging in image-guided radiotherapy: the new standard in ontreatment imaging," International Journal of Radiation Oncology Biology Physics, vol. 64, no. 2, pp. 625-634, 2006.

[9] M. Palombarini, S. Mengoli, P. Fantazzini, C. Cadioli, C. Degli Esposti, and G. P. Frezza, "Analysis of inter-fraction setup errors and organ motion by daily kilovoltage cone beam computed tomography in intensity modulated radiotherapy of prostate cancer," Radiation Oncology, vol. 7, no. 1, article 56, 2012.
[10] J. Boda-Heggemann, F. M. Köhler, H. Wertz et al., "Intrafraction motion of the prostate during an IMRT session: a fiducial-based 3D measurement with Cone-beam CT,' Radiation Oncology, vol. 3, no. 1, article 37, 2008.

[11] R. Sripadam, J. Stratford, A. M. Henry, A. Jackson, C. J. Moore, and P. Price, "Rectal motion can reduce CTV coverage and increase rectal dose during prostate radiotherapy: a daily conebeam CT study," Radiotherapy \& Oncology, vol. 90, no. 3, pp. 312-317, 2009.

[12] J. A. Hatton, P. B. Greer, C. Tang et al., "Does the planning dose-volume histogram represent treatment doses in imageguided prostate radiation therapy? Assessment with cone-beam computerised tomography scans," Radiotherapy and Oncology, vol. 98, no. 2, pp. 162-168, 2011.

[13] T. P. Hellebust, E. Dale, A. Skjønsberg, and D. R. Olsen, "Inter fraction variations in rectum and bladder volumes and dose distributions during high dose rate brachytherapy treatment of the uterine cervix investigated by repetitive CT-examinations," Radiotherapy and Oncology, vol. 60, no. 3, pp. 273-280, 2001.

[14] R. Takam, E. Bezak, E. E. Yeoh, and L. Marcu, "Assessment of normal tissue complications following prostate cancer irradiation: comparison of radiation treatment modalities using NTCP models," Medical Physics, vol. 37, no. 9, pp. 5126-5137, 2010.

[15] X. Allen Li, M. Alber, J. O. Deasy et al., "The use and QA of biologically related models for treatment planning: short report of the TG-166 of the therapy physics committee of the AAPM," Medical Physics, vol. 39, no. 3, pp. 1386-1409, 2012.

[16] J. H. Siewerdsen and D. A. Jaffray, "Cone-beam computed tomography with a flat-panel imager: magnitude and effects of X-ray scatter," Medical Physics, vol. 28, no. 2, pp. 220-231, 2001.

[17] S. Yoo and F. Yin, "Dosimetric feasibility of cone-beam CTbased treatment planning compared to CT-based treatment planning," International Journal of Radiation Oncology Biology Physics, vol. 66, no. 5, pp. 1553-1561, 2006.

[18] Y. Yang, E. Schreibmann, T. Li, C. Wang, and L. Xing, "Evaluation of on-board $\mathrm{kV}$ cone beam CT (CBCT)-based dose calculation," Physics in Medicine and Biology, vol. 52, no. 3, pp. 685-705, 2007.

[19] J. Yang, J. Li, L. Chen et al., "Dosimetric verification of IMRT treatment planning using Monte Carlo simulations for prostate cancer," Physics in Medicine and Biology, vol. 50, no. 5, pp. 869878, 2005.

[20] P. A. Kupelian, K. M. Langen, O. A. Zeidan et al., "Daily variations in delivered doses in patients treated with radiotherapy for localized prostate cancer," International Journal of Radiation Oncology Biology Physics, vol. 66, no. 3, pp. 876-882, 2006.

[21] J. M. Pawlowski, E. S. Yang, A. W. Malcolm, C. W. Coffey, and G. X. Ding, "Reduction of dose delivered to organs at risk in prostate cancer patients via image-guided radiation therapy," International Journal of Radiation Oncology Biology Physics, vol. 76, no. 3, pp. 924-934, 2010.

[22] A. Niemierko, "A generalized concept of equivalent uniform dose (EUD)," Medical Physics, vol. 26, p. 1100, 1999.

[23] J. Y. Park, J. W. Lee, J. B. Chung et al., "Radiobiological modelbased bio-anatomical quality assurance in intensity-modulated radiation therapy for prostate cancer," Journal of Radiation Research, vol. 53, no. 6, pp. 978-988, 2012. 


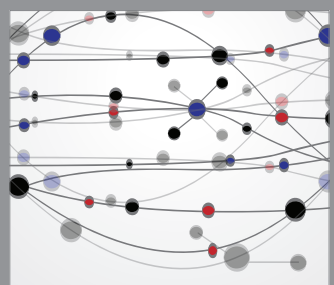

The Scientific World Journal
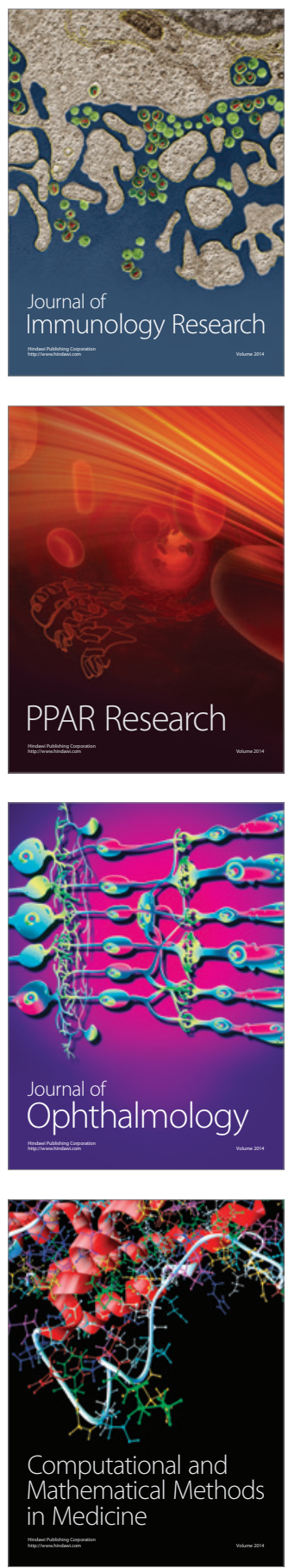

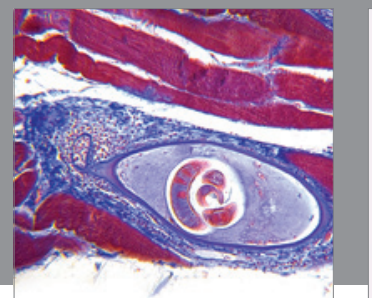

Gastroenterology

Research and Practice
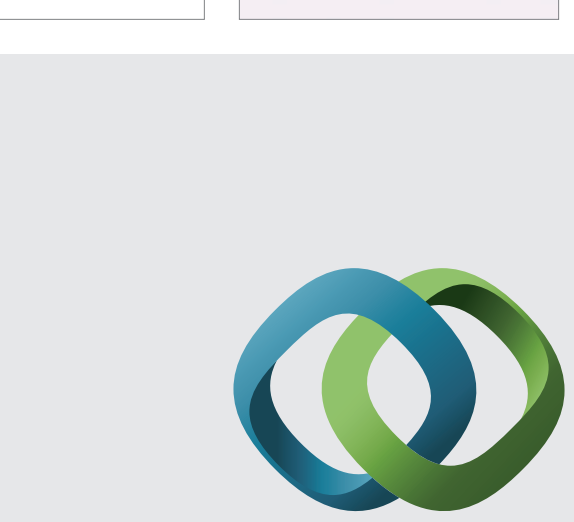

\section{Hindawi}

Submit your manuscripts at

http://www.hindawi.com
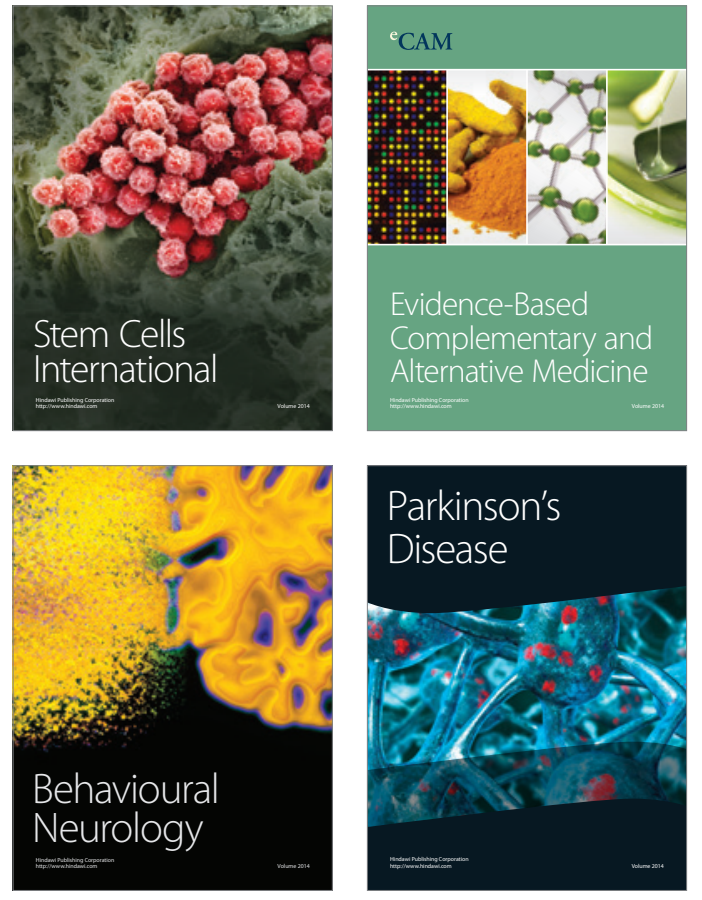
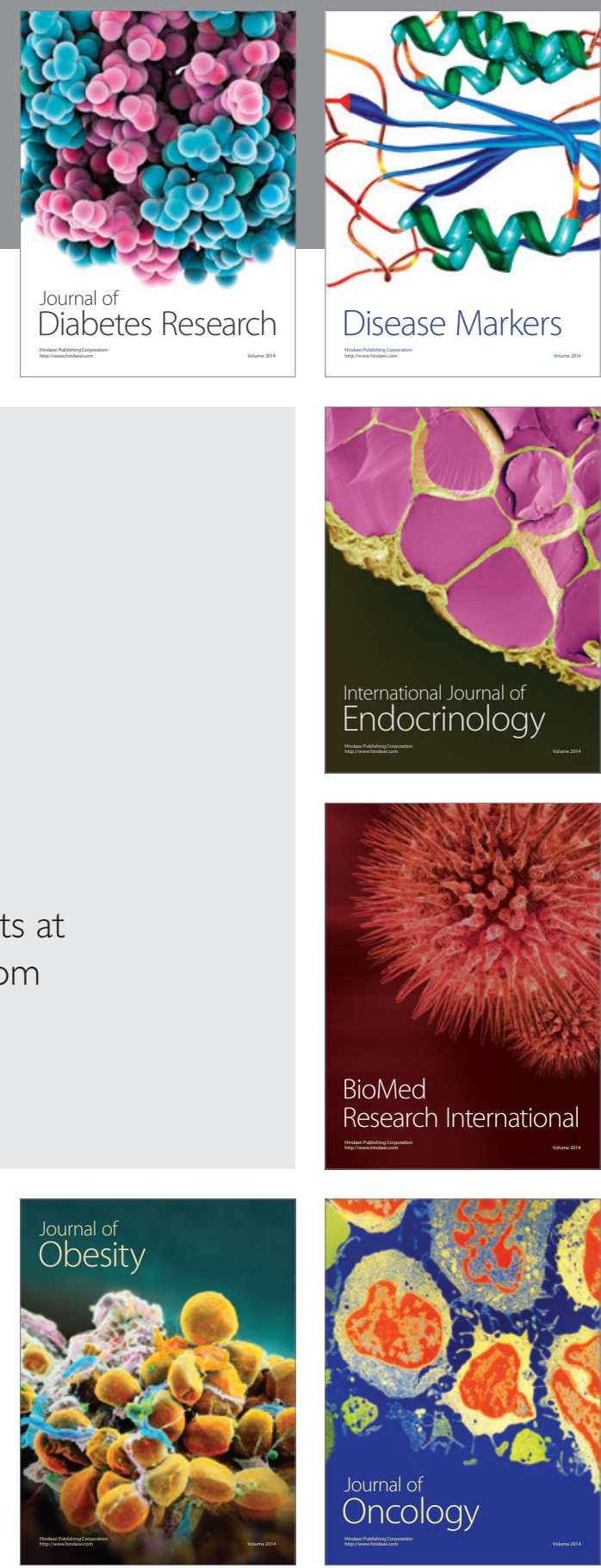

Disease Markers
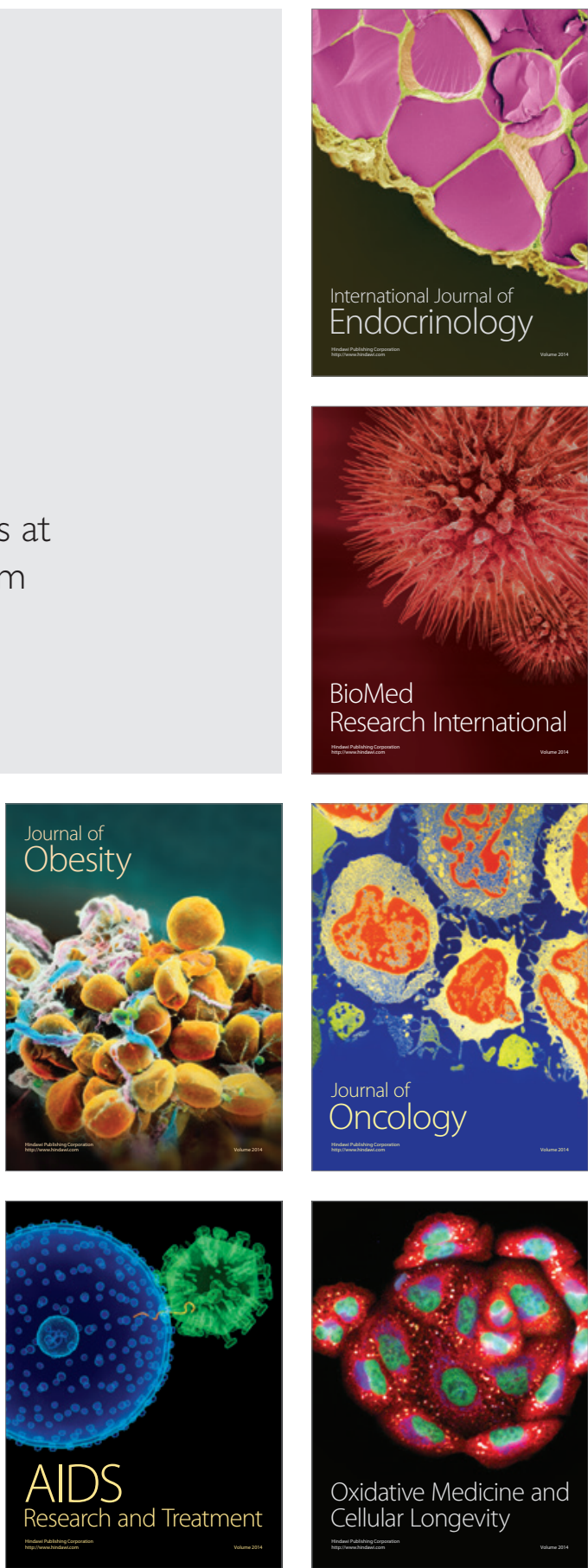